\title{
Diferencias en el desarrollo de Panonychus citri (McGregor) sobre hojas de diversas especies de cítrico (Acarina: Tetranychidae)
}

\author{
FJ Beitia Crespo, A Garrido Vivas \\ Departamento Protección Vegetal, Instituto Valenciano Investigaciones Agrarias, \\ Apartado Oficial, 46113-Moncada (Valencia), España
}

(Recibido el 12 de Agosto de 1989; admitido el 16 de Enero de 1991)

\begin{abstract}
Resumen - Se ha estudiado el desarrollo de $P$ citri (McGregor) sobre hojas de 6 especies de cítrico, para determinar la influencia de la alimentación en la duración de los estados evolutivos y la puesta del ácaro. La experiencia se efectuó con hojas de: naranjos Navel y Valencia (dos variedades de Citrus sinensis), mandarino Clementino ( $C$ clementina), mandarino Satsuma ( $C$ unshiu), limonero (C limon) y pomelo ( $C$ paradisi). Dichas hojas se tomaron en dos períodos del año: mayo y octubre. No se encontraron diferiencias significativas en el tiempo de desarrollo de $P$ citri, según la especie de cítricio utilizada en su alimentación, ni tampoco según la época de recogida de las hojas. Sin embargo, si se han observado diferencias en la puesta del ácaro, pudiendo agruparse las 6 especies, por parejas y en sentido decreciente de puesta, como sigue: limonero y pomelo, Satsuma y Clementino, Navel y Valencia. También se encontró que la puesta es mayor, para todas las especies de cítrico, sobre hojas recogidas en otoño.
\end{abstract}

\section{Panonychus citri / distinta alimentación / desarrollo}

Résumé - Différences du développement de Panonychus citri (McGregor) sur des feuilles de plusieurs espèces d'agrumes (Acarina: Tetranychidae). Le développement de P citri (McGregor) a été étudié sur des feuilles de 6 espèces de Citrus, pour déterminer l'influence de la nourriture sur la durée des stades évolutifs et la ponte des acariens. L'expérience a été réalisée avec des feuilles de : orangers Navel et Valencia (deux variétés de Citrus sinensis), mandarinier Clementino ( $\mathrm{C}$ clementina), mandarinier Satsuma ( $\mathrm{C}$ unshiu), citronnier ( $\mathrm{C}$ limon) et pamplemoussier ( $\mathrm{C}$ paradisi). Les feuilles ont été collectées à deux différentes époques de l'année : mai et octobre. On n'a pas trouvé de différences de développement de $\mathrm{P}$ citri, selon l'espèce de Citrus utilisée et l'époque de ramassage des feuilles (tabl I et II). Par contre, on a observé des différences dans la ponte, et on peut grouper les 6 espèces de Citrus, par couples et en sens décroissant de ponte : citronnier et pamplemoussier, Satsuma et Clementino, Navel et Valencia. La ponte est aussi plus grande, pour toutes les espèces étudiées, sur des feuilles ramassées en automne (tabl III et IV).

\section{Panonychus citri / différentes alimentations / développement}

Summary - Differences in the development of Panonychus citri (McGregor) on leaves of several citrus species (Acarina: Tetranychidae). The development of $\mathrm{P}$ citri (McGregor) on leaves of Citrus plant species has been studied to determine the influence of feeding on duration of evolutive stages and oviposition of mites. Leaves of Navel and Valencia oranges (varieties of Citrus sinensis), Clementino mandarin (C clementina), Satsuma mandarin (C unshiu), lemon (C limon) and grapefruit (C paradisi) were used for the study. Leaves were collected at 2 periods of the year: May and October. Differences in development span of $\mathrm{P}$ citri were observed neither between citrus species nor between the 2 periods of sample collection (tables $I$ and II). However, differences in oviposition were found in decreasing order, in: lemon and grapefruit, followed by Satsuma and Clementino, and then Navel and Valencia. Higher oviposition for all species was also detected on the October leaves (tables III and IV).

Panonychus citri / different food / development 


\section{INTRODUCCION}

En general, los ácaros se dispersan pasivamente gracias al efecto del viento. Según Byrne et al (1982), el proceso de selección de una planta por parte de un ácaro debe contemplarse como una aceptación de la misma para su alimentación, y no como una elección activa por su parte.

De aqui que, si bien $P$ citri sólo constituye una plaga de los cítricos (Van de Vrie et al, 1972; Metcalf \& Flint, 1981), también se le ha encontrado sobre otro tipo de plantas como peral, melocotonero, manzano y almendro, aunque sobre ellas produce poco daño (Shinkaji, 1979; Andrews \& Barnes, 1981; Pena et al, 1984; Hoy, 1985). En España sólo se le ha localizado sobre plantas del género Citrus (Garrido et al, 1984).

Su desarrollo sobre las distintas especies de cítrico es una cuestión que no está bien definida. En general, parece que se desarrolla mejor sobre limonero y pomelo, que naranjo y mandarino (Boyce, 1936; French \& Hutchinson, 1980; Del Rio, 1983, 1986). En España, según Garcia Mari \& Santaballa (1985), P citri se localiza principalmente en naranjo dulce (variedades del grupo Navel) y aunque Clementino y Satsuma también son atacados, las poblaciones que aquí se desarrollan son de menor importancia y los daños producidos inferiores.

En el presente trabajo se han estudiado 6 especies de cítrico, para detectar las diferencias que se producen en el desarrollo y biologia de $P$ citri, en función de su distinta alimentación.

\section{MATERIAL Y METODOS}

Los ejemplares de $P$ citri provenían de una cría de laboratorio sobre limones verdes, mantenida en una cámara climática con condiciones ambientales de $25 \pm$ $2{ }^{\circ} \mathrm{C}$ de temperatura, $65 \pm 5 \%$ de humedad relativa y un fotoperíodo de 18:6 (L:O).

El material vegetal fué hojas de 6 especies de cítrico de una parcela experimental, sometidas al mismo régimen de riego, abonado y demás prácticas culturales. Estas especies eran: naranjo Navel y Valencia (ambas variedades de la especie Citrus sinensis (L) Osb), mandarino Clementino ( $C$ clementina Hort ex Tan), mandarino Satsuma ( $C$ unshiu (Mak) Marc), Limonero (C limon (L) Burm f) y pomelo ( $C$ paradisi Macf).

Las hojas se recogían junto con una porción de rama, que servían para la introducción de la hoja en un tubo de ensayo que contenía una solución nutritiva
(Moutous \& Fos, 1973), lo que aseguraba el mantenimiento de la hoja en buenas condiciones. Un trozo de algodón hidrófilo constantemente humedecido, alrededor del pecíolo, evitaba la fuga de los ácaros presentes en la hoja.

Cada tres días se cambiaban las hojas para proporcionar a los ácaros un alimento óptimo. Se establecieron dos grupos: uno con hojas recogidas en el mes de mayo y otro con hojas de octubre. En ambos casos se tomó la última brotación del año anterior.

\section{Desarrollo experimental}

Se efectuaron dos tipos de experiencias. Una que contempló la duración de los estados de desarrollo de $P$ citri, y otra que estudió la fecundidad del ácaro.

En el primer caso, tras la obtención de una puesta homogénea de 10 huevos/hoja (en una intervalo de 8 horas), que procedía de hembras fecundadas, se seguia la evolución de los mismos hasta el estado adulto, observando la mortalidad que se producía en cada estado evolutivo.

En la segunda experiencia se partía de dos hembras deutoninfa/hoja, que eran fecundadas al llegar al estado adulto, y de las que se apreció la duración de los periodos de puesta y el número de huevos/ hembra.

Se realizaron 10 repeticiones ( 10 hojas) por cada especie de cítrico y tres controles diarios de ambas experiencias.

\section{RESULTADOS}

\section{Desarrollo}

No se observan diferencias significativas en la duración de los estados de desarrollo de $P$ citri, entre las especies de cítrico consideradas; $y$ tampoco se detecta entre los dos grupos de hojas establecidos (tablas I y II).

Por tanto, puede considerarse que el desarrollo del ácaro no se ve afectado por las características de diferentes especies de cítrico, ni por la época del año considerada, factor este que puede determinar una diferente calidad nutricional de un mismo tipo de hoja.

No obstante, cabe destacar la menor mortalidad de estados inmaduros, que se produce cuando la alimentación se realiza sobre Limonero $e$, igualmente para todas las especies, sobre hojas de otoño. 
Tabla I. Duración (en días) de los estados de desarrollo de $P$ citri sobre diferentes especies de cítrico (hojas de otoño). Entre los valores de cada columna no existen diferencias significativas (Anova y test MDS).

\begin{tabular}{lllll}
\hline Especie & Huevo & Larva & Ninfa & Adulto \\
& & & & \\
\hline Navel & $5.63 \pm 0.23$ & $1.90 \pm 0.15$ & $3.51 \pm 0.16$ & $13.65 \pm 1.00$ \\
Valencia & $5.48 \pm 0.22$ & $1.89 \pm 0.14$ & $3.45 \pm 0.13$ & $12.30 \pm 0.90$ \\
Satsuma & $5.44 \pm 0.19$ & $1.83 \pm 0.11$ & $3.60 \pm 0.17$ & $13.70 \pm 0.90$ \\
Clementino & $5.43 \pm 0.15$ & $1.76 \pm 0.14$ & $3.52 \pm 0.18$ & $12.35 \pm 0.76$ \\
Limonero & $5.52 \pm 0.20$ & $1.86 \pm 0.16$ & $3.45 \pm 0.16$ & $14.00 \pm 0.85$ \\
Pomelo & $5.60 \pm 0.20$ & $1.89 \pm 0.12$ & $3.50 \pm 0.13$ & $13.20 \pm 0.95$ \\
& & & & \\
\hline
\end{tabular}

Tabla II. Duración (en días) de los estados de desarrollo de $P$ citri sobre diferentes especies de cítrico (hojas de primavera). Entre los valores de cada columna no existen diferencias significativas (Anova y test MDS).

\begin{tabular}{lcccc}
\hline Especie & Huevo & Larva & Ninfa & Adulto \\
\hline Navel & & & & \\
Valencia & $5.51 \pm 0.13$ & $1.79 \pm 0.10$ & $3.81 \pm 0.11$ & $12.65 \pm 0.72$ \\
Satsuma & $5.41 \pm 0.12$ & $1.84 \pm 0.08$ & $3.82 \pm 0.12$ & $11.25 \pm 0.60$ \\
Clementino & $5.57 \pm 0.21$ & $1.90 \pm 0.09$ & $3.83 \pm 0.13$ & $12.15 \pm 0.54$ \\
Limonero & $5.44 \pm 0.16$ & $1.80 \pm 0.07$ & $3.80 \pm 0.12$ & $11.97 \pm 0.60$ \\
Pomelo & $5.50 \pm 0.11$ & $1.74 \pm 0.09$ & $3.67 \pm 0.13$ & $12.72 \pm 0.64$ \\
& $5.63 \pm 0.15$ & $1.75 \pm 0.08$ & $3.73 \pm 0.10$ & $12.66 \pm 0.69$ \\
\hline
\end{tabular}

\section{Oviposición}

Se ha encontrado que existen diferencias significativas en la puesta de $P$ citri, entre las diversas especies de cítrico (tablas III y IV). Se comprueba que las 6 especies estudiadas pueden agruparse por parejas: Limonero y Pomelo, Satsuma Clementino, Navel y Valencia, en orden decreciente de puesta. Igualmente, para cada especie existen diferencias significativas de la puesta entre las dos épocas de recogida de hojas, observándose una mayor oviposición sobre hojas de otoño.

Cabe pensar que la capacidad de reproducción de la hembra de $P$ citri está determinada por la especie de cítrico de que se alimenta. Igualmente, la distinta composición nutricional de las hojas, dependiendo de la época del año, también parece afectar significativamente esa capacidad de puesta del ácaro.

Tabla III. Duración (en días) de los períodos de vida de la hembra adulta y número de huevos por hembra, en función de la especie de cítrico (hojas de otoño). Los valores de las tres primeras columnas no presentan diferencias significativas. En la última columna, los valores seguidos de letra distinta presentan diferencias significativas (Anova y test MDS).

\begin{tabular}{|c|c|c|c|c|}
\hline \multirow{2}{*}{ Especie } & \multicolumn{3}{|c|}{ Período } & \multirow{2}{*}{ Huevos/Hembra } \\
\hline & Preovip & Oviposición & Postovip & \\
\hline Navel & $1.00 \pm 0.15$ & $11.35 \pm 1.20$ & $1.30 \pm 0.20$ & $31.80 \mathrm{a} \pm 2.30$ \\
\hline Valencia & $1.05 \pm 0.12$ & $9.90 \pm 0.75$ & $1.35 \pm 0.16$ & $30.00 \mathrm{a} \pm 2.49$ \\
\hline Satsuma & $1.20 \pm 0.19$ & $11.10 \pm 0.60$ & $1.40 \pm 0.10$ & $40.70 \mathrm{~b} \pm 1.90$ \\
\hline Clementino & $1.10 \pm 0.11$ & $9.90 \pm 0.90$ & $1.35 \pm 0.14$ & $38.90 \mathrm{~b} \pm 2.12$ \\
\hline Limonero & $1.10 \pm 0.10$ & $11.50 \pm 0.90$ & $1.40 \pm 0.13$ & $46.80 \mathrm{c} \pm 2.22$ \\
\hline Pomelo & $1.00 \pm 0.09$ & $10.86 \pm 1.10$ & $1.34 \pm 0.11$ & $45.70 c \pm 1.95$ \\
\hline
\end{tabular}


Tabla IV. Duración (en días) de los periodos de vida de la hembra adulta y número de huevos por hembra, en función de la especie de cítrico (hojas de primavera). Los valores de las tres primeras columnas no presentan diferencias significativas. En la última columna, los valores seguidos de letra distinta presentan diferencias significativas (Anova y test MDS).

\begin{tabular}{|c|c|c|c|c|}
\hline \multirow{3}{*}{ Especie } & \multirow[b]{3}{*}{ Preovip } & \multicolumn{2}{|c|}{ Período } & \multirow{3}{*}{ Huevos/Hembra } \\
\hline & & & & \\
\hline & & Oviposición & Postovip & \\
\hline Navel & $1.55 \pm 0.20$ & $9.30 \pm 0.85$ & $1.80 \pm 0.18$ & $12.30 a \pm 1.14$ \\
\hline Valencia & $1.61 \pm 0.19$ & $7.80 \pm 1.00$ & $1.84 \pm 0.10$ & $10.70 a \pm 1.08$ \\
\hline Satsuma & $1.70 \pm 0.14$ & $8.45 \pm 1.00$ & $2.00 \pm 0.17$ & $16.90 b \pm 1.58$ \\
\hline Clementino & $1.75 \pm 0.15$ & $8.22 \pm 0.70$ & $2.00 \pm 0.20$ & $15.30 b \pm 1.33$ \\
\hline Limonero & $1.55 \pm 0.11$ & $9.27 \pm 0.95$ & $1.90 \pm 0.09$ & $28.10 c \pm 1.34$ \\
\hline Pomelo & $1.40 \pm 0.14$ & $9.56 \pm 1.00$ & $1.70 \pm 0.13$ & $30.30 c \pm 1.70$ \\
\hline
\end{tabular}

\section{DISCUSION}

Los resultados obtenidos, en cuanto a desarrollo del ácaro, determinan el nulo efecto que tiene la especie de cítrico en la duración de los estados evolutivos; así como la época del año, consideración esta última ya señalada en trabajos previos de otros autores (Costa Comelles et al, 1986), sobre hojas de limonero.

Respecto á la oviposición, la mayor puesta sobre Limonero y Pomelo está de acuerdo con las indicaciones de diversos autores, respecto de la posible preferencia del ácaro observada en campo (Watson \& Berger, 1932; Boyce, 1936; French \& Hutchinson, 1980; Del Rio, 1986). Podría considerarse como la causa de esta preferencia, la composición nutricional de las hojas de cítrico, fundamentalmente a nivel de compuestos nitrogenados y azúcares, que son elementos primordiales en la alimentación de $P$ citri (Fritzsche, 1961). No obstante, Hare (1988) indica que entre limonero y mandarino Satsuma no existe una marcada diferencia en la puesta de $P$ citri, salvo sobre hojas de otoño en las que parece declinar la calidad del mandarino.

Como consecuencia de esta mayor puesta, se obtuvo el valor más alto de tasa intrínseca de desarrollo $\left(r_{m}\right)$ en Limonero y Pomelo, frente a las demás especies que presentan valores parecidos entre ellas. Este fenómeno también se ha observado en Tetranychus cinnabarinus (Bois), quien presenta variaciones de su tasa intrínseca de crecimiento según la planta huésped y con condiciones ambientales semejantes (Gerson \& Aronowitz, 1980).

Por otra parte, se halló un valor superior de $r_{m}$ para los ácaros que se habían desarrollado sobre hojas de otoño, debido igualmente a la puesta, y que lleva a pensar en un mejor desarrollo de las poblaciones de $P$ citri que en primavera, siempre que las condiciones ambientales sean favorables. No obstante, hay que considerar también la gran influencia de la edad y consistencia de las hojas (Henderson \& Holloway, 1942; Costa Comelles et al, 1986), que puede determinar que este fenómeno no sea evidente en campo, pues los ácaros pueden tomar para su alimentación hojas de diferente brotación.

Hasta tal punto debe considerarse éste último aspecto que, en el trabajo de Hare (1988), se indica que hojas de primavera son preferibles a las de otoño, a diferencia de nuestro trabajo, lo que podría achacarse a que en este caso se usaron hojas muy jóvenes, lo cual puede variar por completo los resultados.

Por tanto, se puede considerar que la influencia que la época del año tiene en la calidad nutricional de las hojas es un aspecto muy complejo, que require de más estudio para llegar a su correcta interpretación.

\section{REFERENCIAS}

Andrews KL, Barnes MM (1981) Spider mites on almond in the Southern San Joaquin Valley of California. Env Ent 72(5), 651-654

Boyce AM (1936) The citrus red mite Paratetranychus citri McG in California, and its control. $J$ Econ Ent 29(1), 125-130

Byrne DM, Guerrero JM, Belloti AC, Gracen VE (1982) Behaviour and development of Mononychellus tanajoa (Acari : Tetranychidae) on resistant and susceptible cultivars of cassava. $J$ Econ Ent 75(5), 824-927 
Costa-Comelles J, Garcia-Mari F, Perragut F, Laborda $R$, Marzal C (1986) Influencia de la época del año y la edad de las hojas de los cítricos en el potencial biótico del ácaro rojo Panonychus citri (McGregor) (Acari:Tetranychidae). Actas VIII Jornadas Asoc Esp Ent, 12-21. Sevilla, octubre, 1986

Del Rio G (1983) Un quadriennio di osservazioni su Panonychus citri in Sardegna. Proc EC-Exp Meet "Integrated control in citrus", 18. Siniscola, Muravera Oct 1982. Commission of the European Communities, Luxembourg

Del Rio G (1986) Studies on citrus red mite in Sardinia. In: Control in citrus groves. (Cavalloro, De Martino, eds) Integrated Pest, Amsterdam, 189-197

French JV, Hutchinson EM (1980) Citrus red mite found in Lower Rio Grande Valley. J Rio Grande Valley Hort Soc 34, 107-114

Fritzsche R (1961) Die wirt-parasit beziehungen zwischen verschieden genusepflauzen und Tetranychus urticae Koch und ihve epidemiologische bedentung. Mitt Biol Bundesanst Land-Forstwirtsch Berl-Dahlem 104, 138-143

Garcia Mari F, Santaballa E (1985) ¿Cómo afecta la nueva plaga del ácaro rojo al cultivo de los cítricos? Agricola Vergel Año IV 37, 28-32

Garrido A, Del Busto T, Taranchon J (1984) Bioecologia y control de Panonychus citri (McGregor) (Acarina:Tetranychidae). Levante Agrícola 249-250, 2643

Gerson U, Aronowitz A (1980) Feeding of the carmine spider mite seven host plant species. Ent Exp Appl $28,109-115$
Hare JD (1988) Egg production of the citrus red mite (Acari:Tetranychidae) on lemon and mandarin orange. Environ Entomol 17(4), 715-721

Henderson CF, Holloway JK (1942) Influence of leaf age and feeding injury on the citrus red mit. $J$ Econ Entomol 35(5), 683-686

Hoy MA (1985) Almonds (California). In: Spider mites. Their biology, natural enemies and control (Helle W, Sabelis MW, eds) Vol 1B, Elsevier Sci Publ Amsterdam, 229-310

Metcalf CL, Flint WP (1981) Insectos destructivos e insectos útiles, sus costumbres y su control. Compañía Editiorial Continental SA Mexico Ed XV, $1208 p$

Moutous G, Fos A (1973) Essais de rhizogénèse chez la feuille de vigne isolée. Rev Zool Agric Path Veg, 27-32

Pena JE, Waddill WH, D'Hair SK (1984) Mites attacking cassava in Southern Florida: damage descriptions and density estimate methods. Fla Entomol $67(1), 141-146$

Shinkaji N (1979) Geographical distribution of the citrus red mite Panonychus citri and european red mite Panonychus ulmi in Japan. Recent Adv Acar Vol 1, 81-87

Van de Vrie M, McMurtry JA, Huffaker CB (1972) Ecology of tetranychid mites and their natural enemies. III. Biology, ecology and pest status and host-plants relations of tetranychids. Hilgardia 41 , 343-432

Watson JR, Berger EW (1932) Citrus insects and their control. Bull 77, Agric Ext Serv, Gainsville, FL, $140 p$ 